\title{
Thromboembolism Prophylaxis
}

National Cancer Institute

\section{Source}

National Cancer Institute. Thromboembolism Prophylaxis. NCI Thesaurus. Code

C116684.

The use of medication to prevent the formation of clots and emboli. 